\title{
Algunos ejemplos prácticos de la contribución de una asignatura de matemáticas de Grado a los ODS
}

\author{
Anna Vidal-Meló \\ Departamento de Matemática Aplicada de la Universitat Politècnica de València, Campus de Gandia, \\ avidal@mat.upv.es.
}

\section{Resumen}

Las matemáticas son una herramienta indispensable para comprender $y$ controlar el mundo que nos rodea y como base de cualquier desarrollo tecnológico, imprescindible para hacer sostenible la vida de la especie en nuestro planeta (FECYT, 2014). En este trabajo se describen varias actividades que pueden ser desarrolladas en asignaturas de Matemáticas de los primeros cursos de Grado y que pueden abordarse siguiendo un Aprendizaje Basado en Proyectos. Estas actividades están planteadas a través de problemas contextualizados, relacionados con varios de los 17 Objetivos de Desarrollo Sostenible (ODS) definidos en la Cumbre de Desarrollo Sostenible de septiembre del 2015.

Los problemas de optimización cuadran perfectamente con el ODS de Producción y consumo responsables. Un ejemplo lo constituye la producción de envases alimenticios, envases que encontramos en nuestras despensas y que no siempre son los más eficientes en cuanto a la cantidad de material utilizado. En el estudio de las Ecuaciones Diferenciales es usual comenzar con el modelo exponencial o de Malthus y el modelo logístico. Ambos modelos se asocian al crecimiento de una población, como puede ser la relacionada con un recurso natural como peces, animales terrestres, árboles, etc. Estos modelos pueden modificarse teniendo en cuenta una cuota o tasa de variación relacionada con la pesca o caza controlada, talas, etc., bien de forma constante o de manera periódica (recolección periódica con tiempos de veda). A través de la cooperación entre estudiantes y con programas de simulación y de resolución aproximada como Matlab, se puede analizar cómo varía la población según las cotas permitidas e investigar el máximo valor plausible de forma que no afecte al recurso natural, en el sentido de que no lo haga desaparecer. Con estos problemas se abordan los ODS sobre la Vida submarina y la Vida de ecosistemas terrestres. 
Palabras clave: Matemáticas, ODS, simulación, optimización, cálculo numérico, ecuaciones diferenciales.

\section{Introducción}

En septiembre de 2015 los estados miembros de la ONU definieron un conjunto de 17 Objetivos de Desarrollo Sostenible (ODS) junto con 169 metas, a cumplirse en el 2030 para poner fin a la pobreza, luchar contra la desigualdad y la injusticia y hacer frente al cambio climático (UN, ODS web). También en España en julio de 2018, el Consejo de Ministros aprobó el plan de acción para la implementación de la Agenda 2030, en el que en particular se recogen las aportaciones realizadas por las universidades. Estas ponen a disposición de la Agenda 2030 recursos y aptitudes para promover el desarrollo humano sostenible a través de su responsabilidad en el ámbito de la educación, desarrollando un pensamiento crítico y con el compromiso de incluir competencias relacionadas con un desarrollo sostenible e inclusivo (Gobierno de España, 2019). En los nuevos títulos universitarios se le exige al alumnado la adquisición de competencias transversales relacionadas con su desarrollo personal, 13 en el caso de los títulos de la Universitat Politècnica de València (UPV). Aunque algunas rozan fines de los ODS (CT-07. Responsabilidad ética, medioambiental y profesional; CT-09 Pensamiento crítico; CT-10 Conocimiento de problemas contemporáneos), no implica el conocimiento por parte de nuestro alumnado de los ODS.

Las matemáticas son una herramienta indispensable para comprender y controlar el mundo que nos rodea y son imprescindibles para hacer sostenible la vida de la especie en nuestro planeta (FECYT 2014). Manuel de León constata en una entrevista que ya que las Matemáticas son modelos que resuelven cualquier cosa, encajan en todos 17 objetivos. Como docente de Matemáticas en los primeros cursos de Grado de la UPV me pregunté a principio del curso 2019-2020, cómo implementar algunos ODS en mis asignaturas, a través de la realización de actividades puntuales que pueden presentarse en la realidad además de dar lugar al conocimiento de estos y algunas de sus metas. Para el ODS-12 Producción y consumo responsables, presentamos un problema simple de optimización, generalizable a problemas más complicados. Posteriormente, relacionadas con los ODS-14 vida submarina y el ODS-15 vida de ecosistemas terrestres, se describe una actividad sobre modelos de población, apoyados en el estudio de las ecuaciones diferenciales. Estas actividades pueden ser desarrolladas en asignaturas de Matemáticas de los primeros cursos de Grado y en nuestro caso han sido planteadas durante el curso 2019-2020 a 17 estudiantes de un segundo curso del Grado en Ingeniería de Sistemas de Telecomunicación, Sonido e Imagen (GISTSI) en una asignatura optativa y a 22 estudiantes del Grado en Tecnologías Interactivas (GTI). Aunque pueden abordarse siguiendo un Aprendizaje Basado en 
Proyectos (ABP), no ha sido nuestro caso, pero en un futuro se pueden modificar y ampliar para utilizar esta metodología. Las actividades se han planteado a través de problemas contextualizados, aunque con un nivel muy simple debido a las características del alumnado al que iba dirigido. Proponemos la realización de las actividades de forma paralela al conocimiento del correspondiente ODS y alguna de sus metas, además de plantearles al alumnado una reflexión sobre el problema social y posibles mejoras.

\section{ODS-12: un problema de optimización}

Actualmente el consumo de recursos naturales está aumentando y el ODS-12 Producción y consumo responsables, pretende hacer más y mejores cosas con menos recursos. Entre las metas de este Objetivo, remarcamos la 12.2, lograr la gestión sostenible y el uso eficiente de los recursos naturales y la 12.5, reducción considerable de la generación de desechos (UN, SDGs web). Uno de los problemas actuales de muchas empresas es la reducción de la huella de carbono, que cuantifica las emisiones de gases de efecto invernadero generadas durante todo el ciclo de vida de los productos. Programas como el "CES EduPack", permiten contrastar diferentes alternativas para reducir la huella de un producto realizando cambios en los materiales, procesos de fabricación, medios de transporte, etc., pudiendo de este modo encontrar la opción más adecuada (Montañés, 2016). La optimización del ahorro de material es algo a tener en cuenta en esta reducción, aunque en realidad sólo aporta una pequeña parte en el cálculo de la huella. En la actividad que se propone se estudia reducir la generación de desechos utilizando la menor cantidad de materia prima para un envase. Se trata de una adaptación de un problema clásico: mínima superficie con volumen constante. En este caso se adaptó un ejercicio de matemáticas básicas (Bescós \& Pena, 2000), para tratar el ODS-12. Para cursos superiores de Grado o Máster, pueden plantearse problemas ambientales más complicados como el problema del transporte que tratan Fernández-Torres et al. (2020), en el que se debe encontrar una solución logística optimizada para distribuir materia prima (desechos humanos) desde áreas de suministro a biodigestores.

\subsection{Actividad: envases con menor material}

Esta actividad se centra en la producción de envases que encontramos en nuestras despensas y que no siempre son los más eficientes en cuanto a la cantidad de material utilizado. La metodología utilizada fue el planteamiento al alumnado del GTI de una situación cotidiana, la producción de envases para refrescos de $33 \mathrm{cl}$, partiendo de la pregunta motriz: ¿Piensas que la industria utiliza los envases de la forma más eficiente y sostenible posible? La tarea que se les propuso (Figura 1) trata de minimizar la superficie 
Algunos ejemplos prácticos de la contribución de una asignatura de matemáticas de Grado a los $O D S$

$S(r, h)=2 \pi r h+2 \pi r^{2}$ de una lata cilíndrica, sabiendo que su volumen es $V=\pi r^{2} h=330 \mathrm{~cm}^{3}$, siendo $\mathrm{r}$ el radio $(\mathrm{cm})$ del cilindro y $\mathrm{h}(\mathrm{cm})$ su altura. El alumnado del GTI comprobó que la lata más sostenible, en cuanto a la cantidad de material utilizado, es aquella cuyo radio es de $\mathrm{r}=3.745 \mathrm{~cm}$ y altura $\mathrm{h}=7.49 \mathrm{~cm}$, construyendo una y comparándola con algunas existentes en el mercado. Problemas de más nivel son el de considerar envases en forma de prismas rectangulares o triangulares.

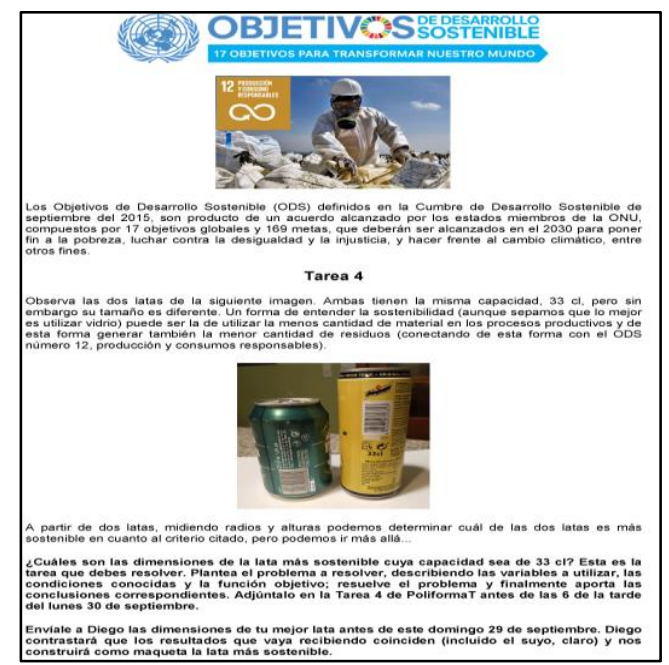

Fig. 1 Tarea de optimización planteada entorno al ODS-12

\section{ODS-14 y 15: modelos poblacionales con Ecuaciones Diferenciales Ordinarias}

En 1798 Thomas Malthus publicó su Ensayo sobre el principio de la población, pronosticando que mientras que la población aumentaba en progresión geométrica, el suministro de comida sólo lo hacía en progresión aritmética, cosa que llevaría a una catástrofe (Wikipedia web). Las guerras, hambrunas y sobre todo la revolución industrial que produjo un cambio total en la producción de alimentos, impidieron esta catástrofe. En el libro Inferno de Dan Brown se plantea también esta amenaza: la sobrepoblación.

En el estudio de la dinámica de poblaciones, dos de los modelos básicos que intentan describir la evolución respecto al tiempo $t$, de una población $y=y(t)$, son el Modelo exponencial o de Malthus y el Modelo Logístico. En el modelo de Malthus, $\frac{d y}{d t}=k y$, se supone que la tasa de nacimientos y muertes son proporcionales a la propia población sin que tengas efecto alguno las limitaciones de espacio o de recursos. El modelo logístico representa una variación del de Malthus, en el sentido de que muchas poblaciones, la 
humana incluida, existen en una cantidad finita de espacio y con recursos limitados, viniendo modelizado por $\frac{d y}{d t}=k y\left(1-\frac{y}{N}\right)$, siendo $N$ la capacidad del sistema o capacidad soporte. Estos modelos pueden modificarse teniendo en cuenta cuotas o tasas de variación relacionada con la repoblación (animal o forestal), recolecciones (talas, pesca, caza), considerando un modelo más general $\frac{d y}{d t}=k y\left(1-\frac{y}{N}\right)+h(t)$ (Universidad de Jaén. Proyecto de innovación docente, 2005-2006). Hay muchos recursos naturales renovables que se utilizan, pero es necesaria una política que permita el máximo uso del recurso sin agotarlo y de forma que se mantenga por encima del nivel adecuado (Zill, 2002, pág. 133). Por ello interesa analizar la evolución de estos recursos, enlazando con los ODS y metas:

- Objetivo 14: Vida submarina. Meta 14.4: reglamentar la explotación pesquera y poner fin a la pesca excesiva ... a fin de restablecer las poblaciones de peces en el plazo más breve posible (UN, SDGs web)

- Objetivo 15: Vida de ecosistemas terrestres. Metas (todas para 2020): 15.1 Velar por la conservación, el restablecimiento y el uso sostenible de los ecosistemas terrestres y de agua dulce...; meta 15.2: Promover la gestión sostenible de todos los tipos de bosques, poner fin a la deforestación, recuperar los bosques degradados e incrementar la forestación y la reforestación a nivel mundial; meta 15.5: Proteger las especies amenazadas y evitar su extinción; meta 15.8: Prevenir la introducción de especies exóticas invasoras y reducir de forma significativa sus efectos en los ecosistemas terrestres y acuáticos ... (UN, SDGs web).

La supervivencia de algunas especies autóctonas puede estar en riesgo por diferentes factores y la repoblación puede garantizar su supervivencia. Con la siguiente actividad se pretendió, entre otras, que el alumnado del GISTSI y del GTI estudiara varios modelos y que decidiera de forma sostenible entre dos acciones de protección y repoblación. Puede variarse el enunciado para animales terrestres, densidad de arbolado, etc.

\subsection{Actividad: Protección y repoblación}

En un pequeño parque natural se desea introducir una especie de pez emblemático en uno de sus lagos, a través de un programa de protección y repoblación. La citada población en el tiempo $t$, medido en meses, viene definida por la función $y=y(t)$, medida en unidades de individuos. Estudia la dinámica de la población de peces, durante el primer año y medio, a través del modelo exponencial $\frac{d y}{d t}=\frac{15}{32} y, y$ a través del modelo logístico 
Algunos ejemplos prácticos de la contribución de una asignatura de matemáticas de Grado a los $O D S$

$\frac{d y}{d t}=\frac{15}{32} y\left(1-\frac{y}{1500}\right)$ para una repoblación inicial de 100 peces. ¿Qué pasaría con el modelo logístico si la repoblación inicial es de 2000? ¿Qué repoblación es preferible?

Si se plantea poder realizar una pesca constante de $C$ peces al mes, el modelo es $\frac{d y}{d t}=\frac{15}{32} y\left(1-\frac{y}{1500}\right)-C$. Estudiar la dinámica para una población inicial de 100 para $C=10,20,30,40, \ldots$, durante el primer año y medio y analizar cuál es la tasa máxima de pesca que puede permitirse para que no desaparezcan los peces.

Aunque en Zill (2002) y otros textos realizan un estudio cualitativo, en nuestro caso solo se ha trabajado a nivel de simulación utilizando Matlab, en prácticas informáticas. Al existir solución analítica puede resolverse con el comando dsolve, obteniendo la evolución (Fig. 2). La Fig. 2 muestra las diferencias entre el crecimiento exponencial y el logístico, debido a que en el de Malthus no hay limitación ni de espacio ni de alimento, cosa que en la realidad suele suceder durante un primer intervalo temporal (a medida que hay más individuos empiezan a aparecer las necesidades de alimento). Para un sistema con capacidad máxima $\mathrm{N}=1500$ individuos, el alumnado debe realizar un razonamiento crítico sobre las diferencias: a pesar de que la tendencia es indiferente del estado inicial (el resultado final a lo largo del tiempo es el mismo, una población tendiendo a 1500), no hay necesidad de mantener inicialmente más individuos de los que pueda aguantar el sistema (más alimento, más agua,...), así pues será suficiente repoblar con 100.
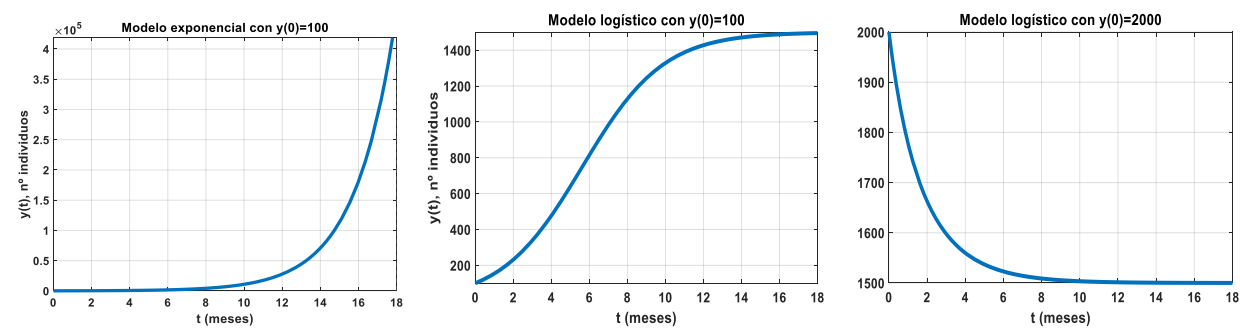

Fig.2 Evolución de la repoblación con 1000 individuos, modelo exponencial y logistico (primera y segunda); evolución de la repoblación con 4000 individuos a través del modelo logístico (última)

Los valores de $\mathrm{C}$ permiten la colaboración entre grupos. A cada grupo de dos estudiantes se les asignó un valor de C. Finalmente englobamos todos los resultados obteniendo que 43 es el máximo valor que permite preservar la población (Figura 3 i.). También puede suponerse captura, o captura y reabastecimiento de forma periódica o estacional, bien a través de una función $h(t)$ definida a trozos (con valores nulos en determinados periodos) o con una función periódica, aunque en este último caso debemos recurrir a métodos de resolución aproximada de ecuaciones diferenciales, como el método de Runge-Kutta con ode45. La 
Fig. 3 (ii y ii) muestra modelos correspondientes a una recolección o captura periódica y a captura y reabastecimiento, observando en las gráficas el efecto de la periodicidad de $h(t)$.
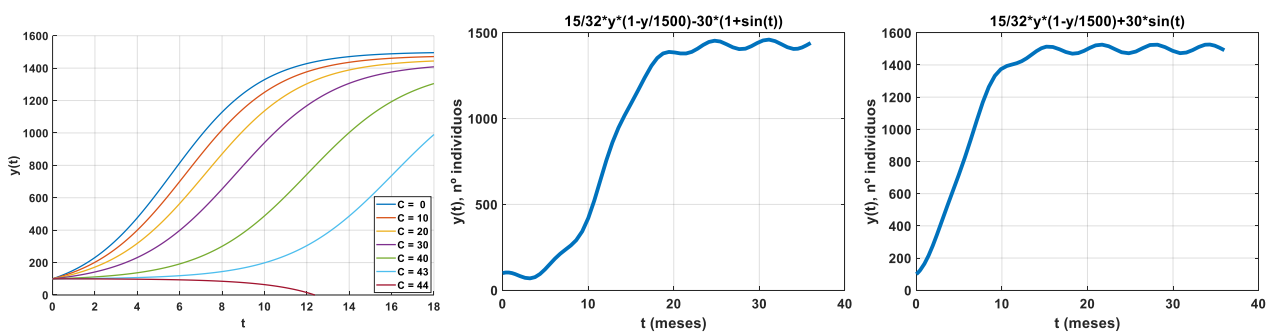

Fig.3: i. Dinámica de la población para diversas pescas constantes C. ii y iii Modelos suponiendo recolección o captura periódica ya captura y reabastecimiento

Pueden estudiarse otros modelos de captura de peces en la web de los Proyectos de Innovación docente de la Universidad de Jaén, además de plantearse actividades relacionadas con los modelos de Lotka-Volterra o el modelo de competición de especies.

\subsection{ODS 3: modelos SIR}

La buena salud es esencial para el desarrollo sostenible y la Agenda 2030 tiene en cuenta la lucha continua contra enfermedades infecciosas. La aplicación de las matemáticas a la epidemiología aparece en 1760 con un tratado sobre la peste europea de Daniel Bernoulli y posteriormente en 1927 con el modelo SIR de Kermack y McKendrick, modelo muy utilizado en el estudio de epidemias (Vidal, Boigues \& Estruch, 2016) y recientemente muy citado en los medios de comunicación para el estudio de la evolución del Covid-19. Desde plataformas como ResearchGate, Google Scholar, Semantic Scholar, repositorios o revistas científicas de libre acceso pueden encontrase artículos relacionados con el modelado de la propagación de virus, estudio de la evolución de un cáncer en función de la dosis de un medicamentos, modelos matemáticos sobre las consecuencias de introducir o no vacunación, simulación del comportamiento epidemiológico, y que pueden servir de base para aplicaciones relacionadas con este ODS (Vidal \& Estruch, 2019)

\section{Conclusiones}

Estas actividades han servido para que el alumnado piense y reflexione de forma crítica respecto a determinados hechos relacionados con los ODS presentes en la actualidad, como la optimización de los recursos naturales. A pesar de que las notas finales de las asignaturas 
implicadas no son malas, no podemos asegurar que las actividades descritas hayan implicado una mejora notable en las notas del alumnado respecto a una docencia más tradicional sin ejemplos prácticos como los expuestos, ya que estas actividades se han planteado y realizado de forma muy puntual. A pesar de ello sí que se ha observado una gran motivación intrínseca del alumnado por este tipo de actividades, atrapando su atención y sintiéndose a gusto con el tema y las actividades realizadas, pidiendo incluso más actividades similares. La intención futura es buscar nuevas actividades para aplicarlas en otros temas del programa, ampliar la implementación de los ODS en nuestras asignaturas y utilizar el ABP siempre que sea posible. También nos resulta interesante poder en próximos cursos recoger la opinión del alumnado respecto a las actividades realizadas y estudiar realmente la efectividad de estas en el proceso enseñanza-aprendizaje.

\section{Agradecimientos}

La autora agradece la ayuda económica y el apoyo institucional recibidos de la Universitat Politècnica de València a través del proyecto PIME 19-20/190.

\section{Referencias}

Bescós, E \& Pena, Z. (2000). Matemáticas-2 Bachillerato . Oxfor Educación.

FECYT (2014). Unidad Didáctica "Matemáticas del Planeta Tierra. SM

Fernández-Torres MJ, Hildebrandt D, Sempuga BC, Caballero JA, Integrating Environmental Concerns into the Teaching of Mathematical Optimization, Education for Chemical Engineers (2020), doi: https://doi.org/10.1016/j.ece.2020.05.005

Gobierno de España (2019). Plan de Acción para la Implementación de la Agenda 2030. Hacia una Estrategia Española de Desarrollo Sostenible. http://www.exteriores.gob.es/Portal/es/SalaDePrensa/Multimedia/Publicaciones/Documents/Area \%20publicaciones/Plan $\% 20 \mathrm{de} \% 20$ Acci $\%$ c3\%b3n\%20para $\% 201 \mathrm{a} \% 20$ Implementacion $\% 20 \mathrm{de} \% 201$ a\%20Agenda\%202030.pdf (consulta Junio 2020).

Montañés Muñoz, N. (2016). Cálculo de la huella de carbono de un envase con ayuda del software CES EduPack. http://hdl.handle.net/10251/68299 (consultado en Junio 2020).

UN, ODS web. https://www.un.org/sustainabledevelopment/es/objetivos-de-desarrollo-sostenible/ (consulta Junio 2020).

Universidad de Jaén. Proyecto de innovacion docente. Coordinacion interdisciplinar de aplicaciones biomatematicas. 2005-2006.

http://ucua.ujaen.es/jnavas/innovacion06_07/cd\%20memoria/cdinnovacion07/cd\%20proyecto/pre senta.html (consulta Junio 2020). 
Vidal Meló, Anna; Estruch, V. D. (2019). El estudio de aplicaciones de los sistemas de ecuaciones diferenciales ordinarias a través de artículos científicos. EN 7th International Conference on Innovation, Documentation and Education (INNODOCT 2019). (671 - 679). Valencia, España: Editorial Universitat Politècnica de València.

Vidal Meló, Anna; Boigues Planes, Francisco José; Estruch, V. D.. (2016) Modelos matemáticos en un problema de epidemias. Modelling in Science Education and Learning, 1 (9), 73 - 85. $10.4995 / \mathrm{msel} .2016 .4426$

Wikipedia web https://es.wikipedia.org/wiki/Cat\%C3\%A1strofe_malthusiana (consulta Junio 2020).

Zill, Dennis G. (2002). Ecuaciones diferenciales con aplicaciones de modelado. México. International Thomson 